\title{
Correction to: Modular Communication as a Structure for Sustainability Within Social Enterprises
}

\author{
Mehak Gupta
}

\section{Correction to:}

Chapter 15 in: A. Chakrabarti et al. (eds.), Design for Tomorrow-Volume 2, Smart Innovation, Systems and Technologies 222, https://doi.org/10.1007/978-981-16-0119-4_15

The original version of the book was published with missed out words in the abstract statement. The chapter and book have been updated with the changes. 